\title{
INDICADORES DE CALIDAD DE LAS \\ PUBLICACIONES CIENTÍFICAS EN PSICOLOGÍA JURÍDICA
}

\author{
QUALITY INDICATORS OF SCIENTIFIC \\ PUBLICATIONS IN LEGAL PSYCHOLOGY
}

\author{
JULIA OSCA-LLUCH ${ }^{1}$, MACARENA TORTOSA ${ }^{2}$, \\ FRANCISCO GONZÁLEZ-SALA ${ }^{3}$ Y FRANCISCO TORTOSA ${ }^{3}$
}

Cómo referenciar este artículo/How to reference this article:

Osca-Lluch, J., Tortosa, M., González-Sala, F. y Tortosa, F. (2017). Indicadores de calidad de las publicaciones científicas en psicología jurídica [Quality Indicators of Scientific Publications in Legal Psychology]. Acción Psicológica, 14(2), 85-98. https://doi.org/10.5944/ap.14.2.20759

\section{Resumen}

Las publicaciones científicas son una de las dimensiones básicas de la evaluación de la calidad de la investigación del personal docente e investigador en España. El criterio más utilizado para la evaluación es la publicación de trabajos en revistas incluidas en las bases de datos de la Web of Science (WoS) o Scopus. El objetivo de este trabajo es conocer que revistas de psicología, que publican trabajos de psicología jurídica, son las que están actualmente incluidas en las bases de datos Web of Science
(WoS) y Scopus, ofrecer un análisis y comparación de los indicadores de calidad de las revistas en estas bases de datos, con el propósito de que los investigadores que trabajan en esta disciplina conozcan la situación de las publicaciones de su campo de conocimiento correspondiente y facilitarles la mejor elección para publicar.

Palabras clave: Revistas científicas; Psicología jurídica; Factor de impacto; Web of Science; JCR; Scopus; SJR.

Correspondencia: Julia Osca Lluch, Instituto de Gestión de la Innovación y del Conocimiento, INGENIO (CSIC-UPV), Universitat Politècnica de València, España. Email: juosllu@ingenio.upv.es

ORCID: Julia Osca Lluch (http://orcid.org/0000-0002-0449-5878), Macarena Tortosa (http://orcid.org/0000-0002-79004877), Francisco González-Sala (http://orcid.org/0000-0003-4124-7459) y Francisco Tortosa (http://orcid.org/0000-0003$\underline{3718-8441)}$.

${ }^{1}$ INGENIO (CSIC-UPV), Universitat Politècnica de València, España.

${ }^{2}$ Universidad Internacional de Valencia-VIU, España.

${ }^{3}$ Universitat de València, España.

Recibido: 05 mayo de 2017.

Aceptado: 18 de julio de 2017. 


\begin{abstract}
The scientific publications are one of the basic dimensions of the evaluation of the quality of research of teaching and research personnel in Spain. The most used criterion for the evaluation is the publication of papers in journals included in the Web of Science (WoS) or Scopus databases. The objective of this work is to know that psychology journals, which publish works of legal psychology, are currently included in the Web of Science (WoS) and Scopus databases, offer an analysis and comparison of the quality indicators of the journals in these databases, in order that researchers working in this discipline know the status of publications in their field of knowledge and provide them with the best choice to publish.
\end{abstract}

Keywords: Scientific journals; Legal psychology; Impact factor; Web of Science; JCR; Scopus; SJR.

\section{Introducción}

Las publicaciones científicas son el principal vehículo de difusión de la ciencia. Su estudio y evaluación está alcanzando un importante desarrollo en los últimos años en las diferentes áreas o disciplinas de la ciencia, por el papel tan relevante que desempeñan en el marco de la investigación científica, como herramientas que resultan fundamentales para la divulgación científica y que, además, posibilitan el avance del conocimiento y alcanzar la excelencia científica. Toda comunidad requiere de un medio de expresión a través del cual registra, transmite e intercambia experiencias entre sus propios miembros y con los de otros grupos. La cultura científica no puede existir al margen de las publicaciones científicas, pero no todas tienen el mismo prestigio y grado de influencia en la comunidad científica. Su reconocimiento depende en gran medida de su calidad y su visibilidad (Osca-Lluch, 2012).

Las revistas científicas definen el "research front" de cualquier disciplina académica. Existe una interacción característica entre nivel de desarrollo institucional e implantación social y número y diversidad de revistas.
Cuando se ofrecen revisiones del estado actual de cualquier ciencia o ámbito de la misma, en general, el protagonismo de los artículos de revista es absoluto. Los revisores recurren a éstas para definir las áreas más importantes y novedosas de investigación y aplicación, y los conceptos, paradigmas, métodos y tecnologías dominantes y definitorias de eso que muchos llaman el "state of art". Incluso es habitual encontrar, en la literatura historiográfica, referencias al singular papel de las revistas en el proceso institucionalizador. Su existencia o inexistencia ayuda a explicar grados de institucionalización y su diversificación es fiel reflejo de la creciente superespecialización que acompaña al desarrollo disciplinar (Quiñones, Peñaranda, López y Tortosa, 2004; Tortosa y Civera, 2001; Tortosa, Santolaya y Civera, 2015). Se puede seguir la actividad de los investigadores o de un departamento o área científica analizando los artículos que publican en revistas científicas (Buela-Casal, Bermúdez, Sierra, Guillén-Riquelme y Quevedo-Blasco, 2015). El análisis de los trabajos da indicaciones sobre el dinamismo de un campo o disciplina científica, sobre su producción científica y sobre los hábitos de publicación y difusión de los investigadores.

Sin embargo, no todas las revistas poseen la misma relevancia como medio para la difusión de la producción investigadora. La escasa visibilidad de las publicaciones científico-técnicas, particularmente de las que se editan en español, afecta a los investigadores cuando son evaluados en el área de sus publicaciones. Así, en este contexto, se observa una tendencia, entre las instituciones y agencias que evalúan la producción de los científicos, a minusvalorar, cuando no a ignorar, las publicaciones de sus trabajos en revistas que no están incluidas en bases de datos internacionales. Por esta razón, la mayor o menor disposición de los autores a publicar en una revista científica, viene influenciada por el grado de visibilidad internacional de la misma, el cual a su vez depende de la presencia en bases de datos bibliográficas internacionales de prestigio.

Uno de los medios para conocer el estado de la actividad investigadora y docente de cualquier institución, consiste en la evaluación de la calidad de las revistas donde publican sus integrantes, aumentando su prestigio en función del factor de impacto que poseen éstas, inde- 
pendientemente del nivel de excelencia del contenido del trabajo publicado (Navarrete-Cortés, Quevedo-Blasco, Chaichio-Moreno, Ríos y Buela-Casal, 2009).

La importancia que la psicología jurídica ha cobrado en los últimos años es un hecho evidente si consideramos su evolución no solo a nivel científico sino también en el terreno aplicado, siendo hoy en día una parte inseparable en el derecho y en la criminología. La Psicología Jurídica ha sido entendida como la aplicación de la ciencia psicológica al Derecho, si bien, tal y como apunta Arce (2005) la Psicología Jurídica no solo cuenta con una parte aplicada, sino que también es capaz de elaborar teorías, metodologías que condicionan las actuaciones propias del Derecho y comprende más allá de éste, al abordar aspectos relevantes y relacionados con el comportamiento humano previo a la comisión de un acto delictivo. Psicología y Derecho aúnan posiciones teóricas, metodológicas y aplicadas para entender los factores propios de las personas, a nivel social, psicológico, conductual, relacional, de salud, entre otros, que afectan o pueden afectar al comportamiento legal de todos los actores que intervienen en el proceso de forma directa como jurados, jueces, peritos, testigos, víctimas, agresores, investigadores, cuerpos de seguridad, o de forma indirecta como grupos de riesgo o la población en general en los estudios relacionados con prevalencia de conductas delictivas, la prevención del riesgo o la influencia de la sociedad, del barrio o de la familia en determinados comportamientos que pueden conducir a un proceso legal (González-Sala, Osca-Lluch, Tortosa y Peñaranda, 2017). Por este motivo, la psicología jurídica tiene un papel fundamental en la toma de decisiones judiciales (Amado, Arce y Fariña, 2015; Amado, Arce, Fariña y Vilariño, 2016; Arce, Fariña y Fraga, 2000) y en la resolución de temas tan lamentables y frecuentes como la violencia doméstica y malos tratos tanto en adultos (Arce, Fariña y Vilariño, 2015; Cala, Trigo y Saavedra, 2016; Contreras y Cano, 2016) como en adolescentes (Álvarez-García, Barreiro-Collazo, Núñez y Dobarro, 2016).

El objetivo de este trabajo es conocer que revistas de psicología, que publican trabajos de psicología jurídica, están actualmente incluidas en las bases de datos Web of Science (WoS) y Scopus y ofrecer un análisis de los in- dicadores de calidad de las revistas en estas bases de datos, con el propósito de que los investigadores que trabajan en esta disciplina conozcan la situación de las publicaciones de su campo de conocimiento correspondiente y facilitarles la mejor elección para publicar.

\section{Material y método}

Este estudio utiliza como fuente de información las bases de datos Journal Citation Reports (Social Sciences Edition y Science Edition), en adelante, JCR, de la empresa Thompson Reuters y el Scimago Journal \& Country Rank (SJR), base de datos que ofrece diversa información e indicadores bibliométricos de las revistas incluidas en la base de datos Scopus, del grupo Elsevier.

Tanto el JCR (SCI y SSCI), como el SJR, clasifican las revistas según una lista de materias que ellos mismos elaboran. En el caso de la psicología, algunas revistas pueden encontrarse indizadas en una, dos o incluso en las tres bases de datos. También pueden estar incluidas en diferentes categorías temáticas, incluso dentro de la misma base de datos, lo que da lugar a que una revista ocupe diferentes posiciones y cuartiles, según la categoría temática en la que este.

Cuando una misma revista se encuentra clasificada en dos o más categorías temáticas diferentes en una base de datos, se considera que esas categorías tienen alguna relación y se puede, a partir de las mismas, detectar relaciones entre las diferentes disciplinas. Estas relaciones existentes entre las diferentes categorías temáticas deberían de tenerse en cuenta, cuando se quieren realizar estudios sobre la producción científica de un grupo de investigación, área o disciplina científica, sobre todo si esta es multidisciplinar, ya que puede haber trabajos de nuestro interés publicados en revistas en categorías temáticas relacionadas con nuestro objeto de estudio.

En este trabajo, a partir de las revistas incluidas en la edición del 2015 de los JCR y SJR, se ha realizado un estudio bibliométrico sobre las revistas de psicología jurídica, que están recogidas en estas bases de datos, con el propósito de conocer que revistas están mejor posiciona- 
Tabla 1

País de edición y solapamiento entre las bases de datos

\begin{tabular}{|c|c|c|c|}
\hline Países & $\begin{array}{c}N^{\circ} \text { revistas solapadas } \\
\text { (JCR y SJR) }\end{array}$ & $N^{0}$ revistas solo SJR & Total revistas por país \\
\hline Australia & 1 & & 1 \\
\hline Croacia & 1 & & 1 \\
\hline Eslovenia & 1 & & 1 \\
\hline España & 1 & 1 & 2 \\
\hline Estados Unidos & 10 & 1 & 11 \\
\hline Holanda & 1 & & 1 \\
\hline Reino Unido & 11 & 1 & 12 \\
\hline Suiza & 1 & & 1 \\
\hline Total & 27 & 3 & 30 \\
\hline
\end{tabular}

das, dentro de esta disciplina, su posición, así como que países lideran la edición de revistas de psicología jurídica.

Se realizó una búsqueda en las bases de datos, seleccionando en cada una de ellas aquellas revistas que estuvieran clasificadas en alguna de las categorías de psicología y que, además también estuvieran clasificadas en alguna de las siguientes categorías temáticas: "Law", "Criminology \& Penology" o incluyera en el título de la publicación o en la institución editora los términos "Law", "Criminology", "Criminal", "Penology", "Forensic", "Legal". La información sobre las revistas seleccionadas procedente de las bases de datos, se descargaron en una base de datos, diseñada "ad hoc" para este estudio.

\section{Resultados}

\section{Cobertura y solapamiento de revistas entre JCR (SCI y SSCI) y SJR}

La consulta a las diferentes bases de datos consultadas, en su edición publicada en el año 2016 dio como resultado un total de 30 revistas. Su distribución por base de datos arrojó un número de revistas muy similar, en JCR (SCI y SSCI) se recuperaron 27 revistas y en SJR, 30.
De todas las revistas seleccionadas, hay que destacar que 27 de ellas, circulan en las bases de datos JCR y SJR, y que solamente hay tres revistas que únicamente están incluidas en el SJR, que son: American Journal of Forensic Psychology, Anuario de Psicología Jurídica y Journal of Forensic Practice.

Un aspecto que hay que mencionar es que, de las 27 revistas incluidas en el JCR, 11 de ellas no están clasificadas en ninguna de las categorías temáticas de psicología, pero que hemos seleccionado para nuestro estudio porque sí que están incluidas en la base de datos SJR.

\section{Cobertura de las bases de datos según país de edición de las revistas}

Cuando se analiza el país de procedencia de las revistas de psicología jurídica incluidas en las bases de datos JCR y SJR, se observa que están editadas por 8 países. Tal como se muestra en la Tabla 1, Reino Unido y Estados Unidos son los países que editan un mayor número de publicaciones, con 12 y 11 revistas respectivamente. El resto de países, solamente tiene una revista de psicología jurídica en el JCR y, en el caso de España, también una revista en el SJR. 


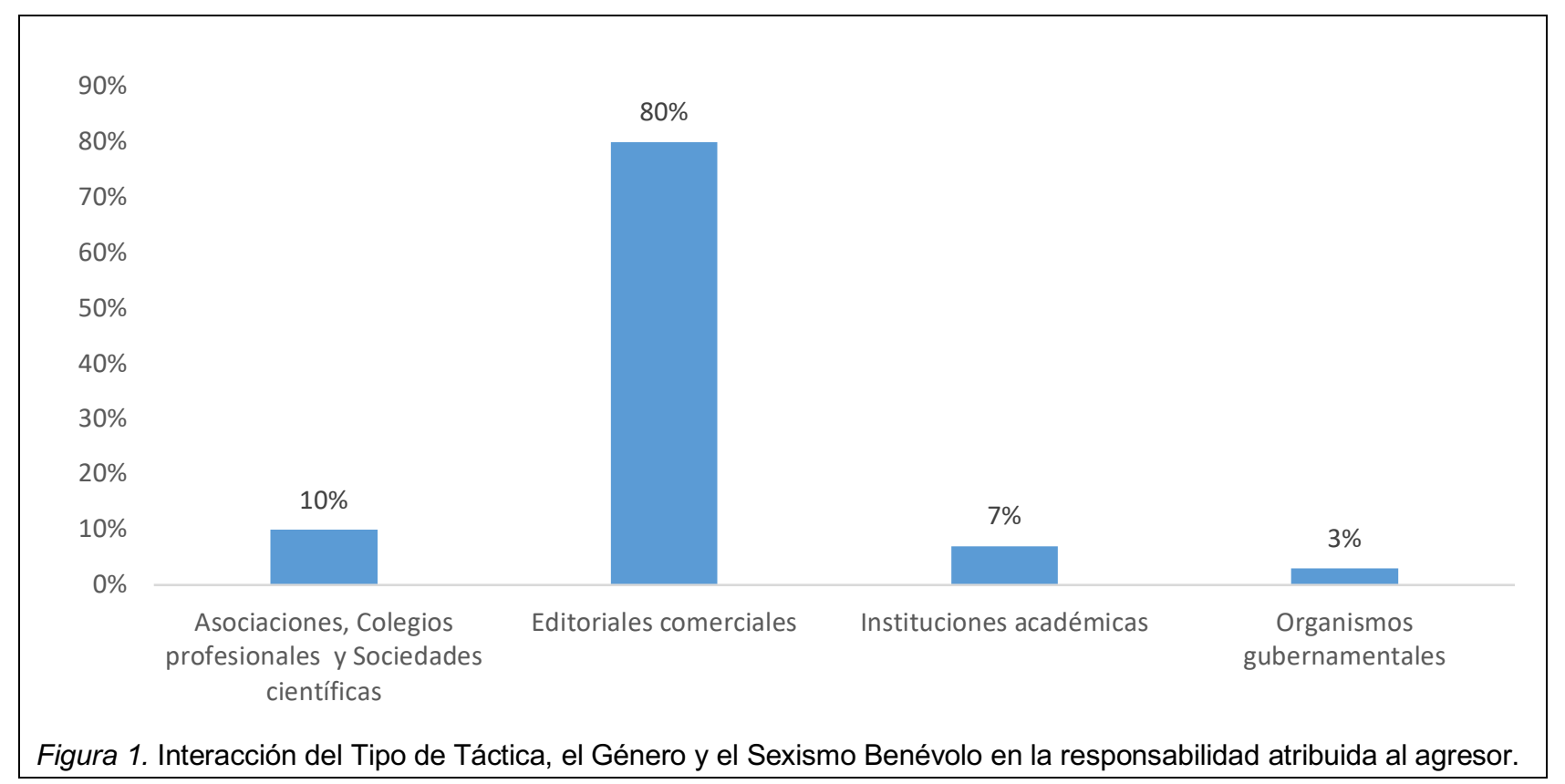

\section{Idiomas de publicación}

La mayor parte de las revistas de psicología jurídica publican sus trabajos en inglés $(90 \%, 27$ revistas). Solamente hay cuatro revistas $(10 \%)$ que publican también en otros idiomas. En este caso se trata de una revista española, que publica en español e inglés, una suiza que publica en francés y, por último, una revista croata y otra eslovena, que publican respectivamente en croata y en esloveno.

\section{Las instituciones editoras de las revis- tas de psicología jurídica}

Cuatro grupos de instituciones publican las revistas de psicología jurídica: las Editoriales comerciales, las Asociaciones, Colegios Profesionales y Sociedades Científicas, las Instituciones académicas y los Organismos gubernamentales. Tal como se muestra en la Figura 1, la mayor parte de las revistas son editadas por editoriales comerciales $(80 \%)$ y Asociaciones, Colegios Profesionales y Asociaciones Científicas (10\%).

\section{Análisis de las categorías temáticas}

Las revistas se han clasificado en 22 categorías temáticas. En JCR, se han clasificado en 10 categorías temáticas y en SJR se han clasificado en 17. Solamente hay cinco categorías temáticas que utilizan tanto las bases de datos JCR como las SJR para clasificar a las revistas analizadas, que son Law, Psychology Applied, Psychology Clinical, Psychology Social y Social Work. Tal como hemos indicado anteriormente, una misma revista, puede estar incluida en varias categorías simultáneamente. En las Tablas 2 y 3 se puede ver la relación de categorías temáticas en las que han sido clasificadas las revistas de psicología jurídica en cada una de las bases de datos. Destaca especialmente Criminology \& Penology en el JCR por aglutinar el mayor número de revistas, mientras que en la base de datos SJR destacan Law, Pathology of Forensic Medicine y Psychology Applied. 
Tabla 2

\section{Categorías temáticas en el JCR}

\begin{tabular}{lc}
\hline Categorías & No $^{\circ}$ revistas \\
\hline Criminology and Penology & 23 \\
Law & 6 \\
Psychology Multidisciplinary & 6 \\
Family Studies & 4 \\
Psychology Applied & 4 \\
Psychology Clinical & 4 \\
Psychiatry & 4 \\
Psychology Social & 2 \\
Social Work & 2 \\
Sociology & 2 \\
\hline
\end{tabular}

Tabla 3

Categorías temáticas en el SJR

\begin{tabular}{lc}
\hline Categorias & $\begin{array}{c}\mathbf{N}^{\mathbf{0}} \\
\text { revistas }\end{array}$ \\
\hline Law & $\mathbf{1 5}$ \\
Pathology of Forensic Medicine & $\mathbf{1 4}$ \\
Psychology Applied & $\mathbf{1 3}$ \\
Psychiatry and Mental Health & $\mathbf{9}$ \\
Psychology Social & $\mathbf{8}$ \\
Psychology (Miscellanous) & $\mathbf{7}$ \\
Psychology Clinical & $\mathbf{5}$ \\
Sociology and Political Science & $\mathbf{4}$ \\
Arts \& Humanities (Miscellaneous) & $\mathbf{3}$ \\
Health (Social Science) & $\mathbf{3}$ \\
Developmental and Education Psychology & $\mathbf{2}$ \\
Social Work & $\mathbf{2}$ \\
Cultural Studies & $\mathbf{1}$ \\
Education & $\mathbf{1}$ \\
Health Professions (Miscellaneous) & $\mathbf{1}$ \\
Medicine (Miscellanous) & $\mathbf{1}$ \\
Public Health, Environmental and & $\mathbf{1}$ \\
Occupational Health & \\
\hline
\end{tabular}

\section{Visibilidad e impacto}

La utilización de indicadores bibliométricos, que cuantifican y analizan la investigación a través de las publicaciones científicas es cada vez más frecuente en la actualidad. Entre los diferentes indicadores utilizado, uno que cada vez tiene mayor influencia es el del factor impacto. La idea de medir el "impacto" producido por un autor, una revista o un grupo en el mundo científico mediante el recuento de las citas que sus publicaciones han tenido en la literatura científica cada vez tiene más trascendencia. Aunque las revistas de "corriente principal" dan mayores garantías al investigador de que su trabajo no pase inadvertido, y aumentan el valor de circulación de los resultados de investigación y les aseguran una mayor cobertura en bases de datos y un mayor potencial de lectores, realmente es el análisis de citas y el factor de impacto los que se ha convertido en el auténtico patrón oro (Torres-Salinas y Cabezas-Clavijo, 2013) en la evaluación de revistas, investigadores, profesores e investigadores, becarios, universidades, proyectos, grupos de investigación y programas de doctorado (Olivas-Ávila y Musi-Lechuga, 2010; Ortiz-de-Urbina-Criado y MoraValentín, 2013; Torres-Salinas, Delgado-López-Cózar, García-Moreno-Torres y Herrera, 2011; Torres-Salinas, Delgado-López-Cózar y Jiménez-Contreras, 2009).

Cada vez es más importante para los investigadores de algunas disciplinas científicas, como la psicología, conocer qué cuartil o posición ocupa una revista dentro de su categoría temática, principalmente, en las bases de datos JCR. La presión por llegar a publicar en las revistas incluidas en los dos primeros cuartiles ha empujado a la falsificación y manipulación de las investigaciones y los currículos de los investigadores. La situación creada ha generado, y no solo en España, una auténtica "cultura de publicación científica en revistas con factor de impacto" en evidente detrimento de la publicación de libros, e incluso ha cambiado el formato tradicional de las tesis doctorales (Buela-Casal, 2014), aunque se trate de una medida altamente polémica (Buela-Casal y Zych, 2012), sin embargo determinar cuáles son las mejores revistas de una determinada área científica o especialidad es una tarea muy compleja (Osca-Lluch, 2005). En la tabla 4 se muestra la relación de revistas de psicología jurídica que actualmente están incluidas en los JCR o SJR de la edición del 2015. Algunas de las revistas de psicología jurídica, que están clasificadas en diferentes categorías temáticas en las dos bases de datos consultadas, ocupan la misma posición en ambas bases de datos y en todas las categorías en las que se encuentran incluidas. Esto sucede en el caso de las revistas Journal of Research in Crime and Delinquency, Journal of Criminal Justice, Journal of Research in Crime and Delinquency, Law and 
Tabla 4

Cuartiles de las revistas de psicología jurídica incluidas en las bases de datos JCR y SJR del año 2015

\begin{tabular}{|c|c|c|c|c|c|c|c|c|}
\hline \multirow{2}{*}{ Revistas } & \multicolumn{4}{|c|}{ JCR } & \multicolumn{4}{|c|}{ SJR } \\
\hline & 2015 & 2015 & 2105 & 2015 & 2105 & 2105 & 2015 & 2105 \\
\hline Aggression and Violent Behavior & Q1 & Q2 & & & Q1 & Q1 & Q1 & \\
\hline American Journal of Forensic Psychology & & & & & Q4 & Q4 & & \\
\hline Anuario de Psicologia Juridica & & & & & Q4 & Q4 & Q4 & \\
\hline Behavioral Sciences and the Law & Q2 & Q3 & & & Q1 & Q1 & Q2 & Q2 \\
\hline British Journal of Criminology & Q1 & & & & Q1 & Q2 & Q2 & Q2 \\
\hline Criminal Behaviour and Mental Health & Q2 & Q3 & & & Q2 & Q2 & Q2 & \\
\hline Criminal Justice and Behavior & Q2 & Q3 & & & Q1 & Q1 & Q1 & \\
\hline Deviance et Societe & Q4 & Q4 & & & Q2 & Q3 & Q3 & Q4 \\
\hline Deviant Behavior & Q3 & Q3 & Q4 & & Q2 & Q1 & Q2 & Q1 \\
\hline $\begin{array}{l}\text { European Journal of Psychology Applied to Legal } \\
\text { Context }\end{array}$ & Q2 & Q3 & & & Q1 & Q3 & & \\
\hline Homicide Studies & Q2 & & & & Q2 & Q2 & Q3 & \\
\hline International Journal of Offender Therapy and & Q2 & Q3 & & & Q2 & Q2 & Q2 & \\
\hline Comparative Criminology & & & & & & & & \\
\hline Journal of Aggression, Maltreatment and Trauma & Q4 & Q4 & Q4 & Q4 & Q2 & Q2 & Q3 & \\
\hline Journal of Criminal Justice & Q1 & & & & Q1 & Q1 & Q1 & Q1 \\
\hline Journal of Forensic Practice & & & & & Q2 & Q3 & Q3 & Q4 \\
\hline Journal of Forensic Psychiatry and Psychology & Q4 & Q4 & & & Q2 & Q3 & & \\
\hline Journal of Forensic Psychology Practice & Q4 & Q4 & & & Q3 & Q4 & & \\
\hline Journal of Interpersonal Violence & Q2 & Q2 & Q2 & & Q1 & Q1 & & \\
\hline Journal of Investigative Psychology and Offender & Q4 & Q4 & & & Q3 & Q3 & & \\
\hline & & & & & & & & \\
\hline Journal of Research in Crime and Delinquency & Q1 & & & & Q1 & & & \\
\hline Law and Human Behavior & Q1 & Q1 & & & Q1 & Q1 & Q1 & Q1 \\
\hline Legal and Criminological Psychology & Q1 & Q1 & Q2 & & Q1 & Q2 & & \\
\hline Ljetopis Socijalnog Rada & Q4 & & & & Q4 & Q3 & Q4 & Q3 \\
\hline Psychiatry Psychology and Law & Q3 & Q3 & Q3 & Q4 & Q2 & Q3 & Q3 & Q3 \\
\hline Psychology of Violence & Q1 & Q1 & Q1 & & Q1 & Q1 & Q1 & \\
\hline Psychology, Crime \& Law & Q2 & Q2 & Q3 & & Q2 & Q1 & Q2 & \\
\hline Revija za Kriminalistiko in Kriminologijo & Q4 & & & & Q4 & Q4 & Q4 & \\
\hline Sexual Abuse-A Journal of Research and Treatment & Q2 & Q1 & & & Q1 & Q1 & & \\
\hline Trauma, Violence and Abuse & Q1 & Q1 & Q1 & & Q1 & Q1 & Q1 & Q1 \\
\hline Youth Violence and Juvenile Justice & Q1 & & & & Q1 & Q1 & Q1 & \\
\hline
\end{tabular}

Human Behavior, Trauma, Psychology Violence, Violence and Abuse y Youth Violence and Juvenile Justice, que están clasificadas en todas las categorías temáticas de las bases de datos en el cuartil 1. Sin embargo, se observa que lo más frecuente ( 24 revistas), es que una revista, ocupe diferentes posiciones diferentes y, por lo tanto, diferente cuartil, dependiendo de la categoría temática en la que ha sido clasificada.

\section{Discusión}

Es apresurado hacer un balance definitivo de la investigación en psicología jurídica en la actualidad, pero siendo optimistas y viendo el estado de las revistas de psicología que publican trabajos de jurídica, podría decirse que la consolidación de la psicología jurídica está en marcha. Este trabajo revela que, aunque no existe uan categoría temática especifica de psicología jurídica en las bases de datos consultadas. Sin embargo, hay suficiente 
número de revistas, que reúnen las condiciones de estar clasificadas en categorías temáticas de psicología y jurídica, que viene a ser un indicador de la consolidación que va teniendo esta disciplina.

De acuerdo con los datos obtenidos, de las 30 revistas que cumplen los criterios en las Bases de Datos consultadas, 27 en JCR (SCI y SSCI) y 30 en SJR, sólo 3 de ellas están fuera de JCR (American Journal of Forensic Psychology, Anuario de Psicología Jurídica y Journal of Forensic Practice). De las 27 revistas incluidas en el JCR, 11 de ellas no están clasificadas en ninguna de las categorías temáticas de psicología, lo que no las tornaría, en principio, atractivas para psicólogos jurídicos y forenses, lo que dejaría en 16 el número potencial de targets para quienes investigan y /o trabajan en el ámbito.

Las 30 revistas se han clasificado en 22 categorías temáticas, una muy elevada dispersión. Las 27 revistas incluidas en JCR se han clasificado en 10 categorías temáticas, y las 30 incluidas en SJR se han clasificado en 17. Solamente hay cinco categorías temáticas que utilizan tanto las bases de datos JCR como las SJR para clasificar a las revistas analizadas, que son Law $(6+15)$, Psychology Applied (4+13), Psychology Clinical (4+5), Psychology Social (2+8) y Social Work (2+2). Criminology and Penology en JCR con 23 revistas y Pathology of Forensic Medicine en SJR con 14 son las categorías propias en las que se clasifica un mayor número de revistas.

Un $80 \%$ de las revistas las publican editoriales comerciales, un $10 \%$ Asociaciones, Colegios Profesionales y Asociaciones Científicas, un $7 \%$ instituciones académicas y un $3 \%$ organismos gubernamentales. Un aspecto que hay que destacar es que, aunque en conjunto, las revistas proceden de ocho países diferentes, solamente dos países son los que editan el $77 \%$ de las publicaciones: Estados Unidos y Reino Unido.

Respecto al idioma de publicación de los trabajos, hay que mencionar que las revistas de lengua materna inglesa son 24 (12 UK, 11 EU y uno Australia), y seis proceden de países europeos con lenguas nativas diferentes (Croacia, Eslovenia, España, Holanda y Suiza), pero dos de ellas, una española y la holandesa también publi- can en inglés. De esta forma, se observa que solo cuatro revistas publican en lengua propia, e incluso una lo hace en español e inglés, una situación que otorga un peso demoledor al inglés, y recuerda la época medieval en la que el latín era la lengua de la cultura y las demás lenguas no existían en el mundo de la ciencia.

Las revistas de mayor atractivo para los investigadores serían sin duda: Journal of Criminal Justice; Journal of Research in Crime and Delinquency; Law and Human Behavior; Psychology of Violence; Trauma, Violence and Abuse; y Youth Violence and Juvenile Justice, clasificadas en todas las categorías temáticas de las bases de datos (JCR y SJR) en el cuartil 1. También sería muy recomendable el British Journal of Criminology (Q1 en JCR y Q1 y Q2 en SJR). No obstante, lo más frecuente (24 revistas de 30 ), es que una revista, ocupe posiciones diferentes y, por lo tanto, distinto cuartil, dependiendo de la categoría temática en la que hubiera sido clasificada.

Sin embargo, del total de las publicaciones analizadas, desde el punto de vista de los criterios de evaluación de la Comisión Nacional Evaluadora de la Actividad Investigadora (CNEAI), encargada de evaluar periodos sexenales de actividad investigadora de los profesores universitarios y del personal de las escalas científicas del $\mathrm{CSIC}^{1}$, solamente 18 revistas de psicología jurídica, de las analizadas en este trabajo, las incluidas en el JCR, servirían para la acreditación de investigadores españoles. Las revistas que cumplen estos requisitos son: British Journal of Criminology (Q1), Journal of Criminal Justice (Q1), Journal of Research in Crime and Delinquency (Q1), Law and Human Behavior (Q1, Q1), Psychology of Violence (Q1, Q1, Q1), Trauma, Violence and Abuse (Q1, Q1, Q1), Youth Violence and Juvenile Justice (Q1), Aggression and Violent Behavior (Q1, Q2), Legal and Criminological Psychology (Q1, Q1, Q2), Sexual Abuse-A Journal of Research and Treatment (Q2, Q1), Behavioral Sciences and the Law (Q2, Q3), Criminal Behaviour and Mental Health (Q2, Q3), Criminal

\footnotetext{
${ }^{1}$ Resolución de 30 de noviembre de 2016, de la Secretaría de Estado de Educación, Formación profesional y Universidades, por la que se fija el procedimiento y plazo de presentación de solicitudes de evaluación de la actividad investigadora a la Comisión Nacional Evaluadora de la Actividad Investigadora. Publicado en: «BOE» núm. 291, de 2 de diciembre de 2016, páginas 84669 a 84674 .
} 
Justice and Behavior (Q2, Q3), European Journal of Psychology Applied to Legal Context (Q2, Q3), Homicide Studies (Q2), International Journal of Offender Therapy and Comparative Criminology (Q2, Q3), Journal of Interpersonal Violence (Q2, Q2, Q2), Psychology, Crime \& Law (Q2, Q2, Q3).

El análisis de las revistas de psicología jurídica demuestra la existencia de una disciplina que, en España, cada día está más consolidada pero que necesita contar con unas revistas especializadas más consolidadas, con el propósito de que estos pueden publicar los resultados de sus investigaciones en revistas nacionales de su especialidad. Actualmente, las pocas opciones que tienen los especialistas de la materia para publicar trabajos en revistas de su disciplina que les sirvan para que se reconozcan sus méritos académicos, contribuye a que estos deriven sus trabajos a revistas de carácter general o multidisciplinar, lo que contribuye a restar visibilidad a los trabajos o que estos pasen desapercibidos para el resto de especialistas en la materia, que consultan para su formación o actualización la información bibliográfica en revistas especializadas.

\section{Referencias}

Álvarez-García, D., Barreiro-Collazo, A., Núñez, J. C. y Dobarro, A. (2016). Validity and Reliability of the Cyber-Aggression Questionnaire for Adolescents (CYBA). European Journal of Psychology Applied to Legal Context, 8, 69-77. https://doi.org/10.1016/j.ejpal.2016.02.003

Amado, B. G., Arce, R. y Fariña, F. (2015). Undeutsch Hypothesis and Criteria Based Content Analysis: A Meta-Analytic Review. European Journal of Psychology Applied to Legal Context, 7, 3-12. https://doi.org/10.1016/j.ejpal.2014.11.002

Amado, B. G., Arce, R., Farina, F. y Vilariño, M. (2016). Criteria-Based Content Analysis (CBCA) Reality Criteria in Adults: A Meta-Analytic Review. International Journal of Clinical and Health Psychology, $16 \quad$ 201-210. https://doi.org/10.1016/j.ijchp.2016.01.002

Arce, R. (2005). La construcción de la psicología jurídica en Europa y su estatus actual [La construcción de la psicología jurídica en Europa y su estatus actual]. En R. Abrunhosa y C. Machado (Eds.), Manual de psicología jurídica (pp. 103-114). Coimbra, Portugal: Quarteto.

Arce, R., Fariña, F. y Fraga, A. (2000). Género y formación de juicios en un caso de violación [Gender and Juror Judgment Making in a Case of Rape]. Psicothema, 12(4), 623-628. Recuperado de http://www.psicothema.com/pdf/381.pdf

Arce, R., Fariña, F. y Vilariño, M. (2015). Daño psicológico en casos de victimas de violencia de género: Estudio comparativo de la evaluación forense [Psychological Injury in Intimate Partner Violence Cases: A Contrastive Analysis of Forensic Measures]. Revista Iberoamericana de Psicología y Salud, 6 , 72-80. 
Buela-Casal, G. (2014). Pathological Publishing: A new Psychological Disorder with Legal Consequences? European Journal of Psychology Applied to Legal Context, 6, 91-97. https://doi.org/10.1016/j.ejpal.2014.06.005

Buela-Casal, G., Bermúdez, M. P., Sierra, J. C., GuillénRiquelme, A. y Quevedo-Blasco, R. (2015). Productividad y eficiencia en investigación por comunidades autónomas españolas según la financiación (2012) [Productivity and Efficiency in Research for Spanish Regions by Funding (2012)]. Revista Iberoamericana de Psicología y Salud, 6, 110. https://doi.org/10.1016/S2171-2069(15)70001-3

Buela-Casal, G. y Zych, I. (2012). What Do the Scientists Think about the Impact Factor? Scientometrics, $\quad 92, \quad 281-292$. https://doi.org/10.1007/s11192-012-0676-y

Cala, J., Trigo, M. E. y Saavedra, F. J. (2016). Women's Disengagement from Legal Proceedings for Intimate Partner Violence: Sociodemographic and Psychological Variables. European Journal of Psychology Applied to Legal Context, 8, 35-42. https://doi.org/10.1016/j.ejpal.2015.10.002

Contreras, L. y Cano, M. D. (2016). Child-to-parent Violence: The Role of Exposure to Violence and its Relationship to Social-Cognitive Processing. European Journal of Psychology Applied to Legal Context, $\quad 8, \quad 43-50$. https://doi.org/10.1016/j.ejpal.2016.03.003

González-Sala, F., Osca-Lluch, J., Tortosa Gil, F. y Peñaranda Ortega, M. (2017). Characterization of Legal Psychology through Psychology Journals included in Criminology \& Penology and Law Categories of Web of Science. Anales de Psicología, 33, 411-416. https://doi.org/10.6018/analesps.33.2.262591

Navarrete-Cortés, J., Quevedo-Blasco, R., ChaichioMoreno, J. A., Ríos, C. y Buela-Casal, G. (2009). Análisis cuantitativo por países de la productividad en psicología de revistas en la Web of Science
[Quantitative Analysis by Country of Psychological Material Published in Journals in the Web of Science]. Revista Mexicana de Psicología, 26, 131$143 . \quad$ Recuperado de http://www.redalyc.org/html/2430/243016315001/

Olivas-Ávila, J. y Musi-Lechuga, B. (2010). Análisis de la producción de los profesores funcionarios de Psicología en España en artículos de revistas de la Web of Science [Analysis of the Production of Psychology Professors in Spain in Journal Articles of the Web of Science]. Psicothema, 22, 909-916. Recuperado de https://www.unioviedo.es/reunido/index.php/PST/ar ticle/viewFile/8970/8834

Ortiz-de-Urbina-Criado, M. y Mora-Valentín, E. (2013). El sistema de acreditación del profesorado a través del Programa ACADEMIA: Evolución y cambios. [The National Accreditation System of University Professors through The ACADEMIA Program: Evolution and Changes]. Revista Española de Documentación Científica, 36(1), 1-8. https://doi.org/10.3989/redc.2013.1.971

Osca-Lluch, J. (2005). Some Considerations on the Use of the Impact Factor of Scientific Journals as a Tool to Evaluate Research in Psychology. Scientometrics, $\quad 65$, 189-197. https://doi.org/10.1007/s11192-005-0267-2

Osca-Lluch, J. (2012). Aspectos regionales de las revistas españolas de ciencias sociales y humanidades: calidad y visibilidad internacional [Regional Aspects of Social Sciences Spanish Journals: Quality and International Visibility]. Biblio 3 W. Revista Bibiográfica de Geografia y Ciencias Sociales, XVII, 998. Recuperado de http://www.ub.edu/geocrit/b3w-998.htm

Quiñones, E., Peñaranda, M., López, J. J. y Tortosa, F. (2004). Social and Personality Psychology as reflected in Authorships and Citations of Journal of Personality and Social Psychology, 1965-2000. Journal of Personality and Social Psychology, 86, 435-452. 
Torres-Salinas, D. y Cabezas-Clavijo, Á. (2013). Cómo publicar en revistas científicas de impacto: Consejos y reglas sobre publicación científica [How to Publish in Impact Scientific Journals: Advice and rules on Scientific Publication]. EC3 Working Papers, 13. Recuperado de https://ec3metrics.com/wpcontent/uploads/2013/07/2-ART\%C3\%8DCULO$\mathrm{C} \% \mathrm{C} 3 \% \mathrm{~B} 3$ mo-Publicar-en-Revistas-

Cient $\%$ C3\%ADficas-de-Impacto_Consejos-yReglas-sobre-Publicaci\%C3\%B3nient $\% \mathrm{C} 3 \%$ ADfica.pdf

Torres-Salinas, D., Delgado-López-Cózar, E., GarcíaMoreno-Torres, J. y Herrera, F. (2011). Rankings ISI de las universidades españolas según campos científicos: Descripción y resultados [ISI Rankings of Universities in Spain by Scientific Field]. El Profesional de la Información, 20(1), 111-122. Recuperado de http://eprints.rclis.org/15325/

Torres-Salinas, D., Delgado-López-Cózar, E. y JiménezContreras, E. (2009). Redes de citación de las revistas españolas de Ciencias Sociales 1994-2006 [Citation Networks among Spanish Social Sciences journals 1994-2006]. Revista Española de Documentación Científica, 32(2), 34-50. https://doi.org/10.3989/redc.2009.2.686

Tortosa, F. y Civera, C. (2001). Revistas y disciplina psicológica. Cien años de encuentro [Journals and Psychological Discipline. One Hundred Years of Encounter]. Papeles del Psicólogo, 79, 3-14. Recuperado de http://www.papelesdelpsicologo.es/resumen?pii=85 8

Tortosa, F., Santolaya, F. y Civera, C. (2015). La psicología española contemporánea. ¿Una realidad pletórica? [The Spanish Contemporary Psychology. An Eventful Reality?] Informaciò Psicològica, 109, 51-71. Recuperado de http://www.informaciopsicologica.info/OJSmottif/i ndex.php/leonardo/article/download/226/407 


\title{
QUALITY INDICATORS OF SCIENTIFIC PUBLICATIONS IN LEGAL PSYGHOLOGY
}

\author{
JULIA OSCA-LLUCH ${ }^{1}$, MACARENA TORTOSA ${ }^{2}$, \\ FRANCISCO GONZÁLEZ-SALA ${ }^{3}$ Y FRANCISCO TORTOSA ${ }^{3}$
}

\section{EXTENDED SUMMARY}

\section{Introduction}

Scientific publications are the main vehicles for the dissemination of science. The study and evaluation of such is reaching an important development in recent years in different areas or disciplines of science, because of the important role that they play within the framework of scientific research, as tools that are essential to the dissemination of science and that they furthermore enable the advancement of knowledge and achieve scientific excellence. Community requires a means of expression through which it records, transmits and exchanges experiences among its own members and those of other groups. Scientific culture cannot exist independently from scientific publications, but not all have the same prestige and degree of influence in the scientific community. Their recognition depends largely on their quality and their visibility (Osca-Lluch, 2012).

There is no doubt that not all journals have the same relevance as a means for the dissemination of the production of research. The limited visibility of scientific and technical publications, particularly of ones which are published in Spanish, affects researchers when they are evaluated in the area of their publications. Thus, in this context, there is a trend among institutions and agencies that evaluate the scientific production, to underestimate, if not ignore, the publications of the work in journals that are not included in international databases. For this reason, the willingness of authors to publish in a particular scientific journal, is influenced by the degree of international visibility of said journal, which in turn depends on its presence in international bibliographic databases of prestige.

One of the ways to learn about the current state of the research and teaching activity of any institution or any scientific and professional profile, consists of the evaluation of the quality of the journals where its members are published, increasing its prestige according to the impact factor these possess, regardless of the level of excellence of the content of the published work.

The objective of this study is to know which journals of psychology, that publish works in legal psychology are currently included in Scopus and Web of Science (WoS) databases and offer an analysis of the indicators of quality of journals in these databases, with the purpose that researchers working in this discipline know the state of publicatios in their field of knowledge and so facilitate the best choice for publishing.

\section{Method}

In this work, from the journals included in the edition of the 2015 of the JCR and SJR, we have conducted a Bibliometric study on forensic psychology journals, which are contained in these databases, with the purpose of finding out which journals are better positioned within this discipline, the position as well as which countries lead the publishing of forensic psychology journals.

We conducted a search in the databases, selecting from each of them those journals that were classified into one of the categories of psychology and were moreover also classified in one of the following thematic categories: "Law", "Criminology \& Penology" or that included the terms "Law", "Criminology", "Criminal", "Penology", "Forensic", "Legal" in the title of the publication or 
the publishing institution. Information about the journals selected from these databases, was downloaded into a database, designed "ad hoc" for this study.

\section{Results}

The consultation of the different databases, in its edition published in the year 2016, resulted in a total of 30 journals. Distribution by database showed a very similar number of journals, JCR (SCI and SSCI) recovered 27 journals and SJR, 30.

For all selected journals, it should be noted that 27 of them circulate in the JCR and SJR databases, and that there are only three journals that are only included in the SJR, which are: American Journal of Forensic Psychology, Yearbook of forensic psychology and Journal of Forensic Practice.

When the country of origin of legal psychology journals included in JCR and SJR database is analyzed, we observed that these journals are published by eight countries. United Kingdom and United States are the countries that publish a greater number of publications, with 12 and 11 journals respectively. Other countries (Australia, Croatia, Slovenia, Spain, the Netherlands and Switzerland), only have 1 journal of forensic psychology in the JCR and, in the case of Spain, also one journal in the JRS.

Most of the forensic psychology journals publish their works in English $(90 \%, 27$ journals). There are only four journals $(10 \%)$ which are also published in other languages. This is a case of a Spanish journal, which publishes in both English and Spanish, a Swiss one which publishes in French and, finally, a Croatian journal and another Slovenian, respectively published in Croatian and Slovenian.

Four groups of institutions publish legal psychology journals: The Commercial publishers, the Associations, Professional Associations and Scientific societies, Academic Institutions and Government agencies. Most of the journals are published by Commercial publishers (80\%) and Associations, Professional Associations and Scientific Associations (10\%).
The journals have been classified into 22 subject categories. In JCR, they have been classified in 10 theme categories and in SJR in 17. There are only five categories that are used by both the SJR and JCR databases for classifying the analysed journals, which are the following, Law, Applied Psychology, Clinical Psychology, Social Psychology, and Social Work. In tables 2 and 3, you can see the subject categories which have been classified in legal psychology journals in each of the databases. Criminology \& Penology comes to the attention in the JCR by bringing together the largest number of journals, while in SJR database it is Law, Pathology of Forensic Medicine and Psychology Applied that bring together the largest number of journals.

The use of Bibliometric indicators, that quantify and analyse research through scientific publications is used increasingly often nowadays. Between the different indicators used, one which has a growing influence is the impact factor. Citation analysis and impact factor, define the criteria for agencies evaluation of journals, researchers, professors and researchers, scholars, universities, projects, research groups and doctoral programs.

Table 4 shows the relationship of legal psychology journals which are currently listed in the JCR or SJR in the 2015 Edition. Some of the journals of forensic psychology, which are classified in different categories in the two databases consulted, occupy the same position in both databases and in all the categories that they are included in. This happens in the case of the journals; Journal of Research in Crime and Delinquency, Journal of Criminal Justice, Journal of Research in Crime and Delinquency, Law and Human Behaviour, Trauma, Psychology Violence, Violence and Abuse and Youth Violence and Juvenile Justice, which are classified in all thematic categories of the databases in quartile 1. However, it appears that the most common ( 24 journals), is for a journal, to occupy different positions and, therefore, a different quartile, depending on the subject category in which it has been classified. 


\section{Discussion}

According to data obtained from 30 journals that meet the criteria in the consulted databases, 27 in JCR (SCI and SSCI) and 30 in JRS, only 3 of them are outside JCR (American Journal of Forensic Psychology, Yearbook of forensic psychology and Journal of Forensic Practice). Of 27 journals listed in the JCR, 11 of them are not classified in any of the thematic categories of psychology, which would not make them, in principle, attractive for legal and forensic psychologists, which would make 16 the potential number of targets for those who investigate and/or work in the field.

The journals of major attraction for researchers would with no doubt be: Journal of Criminal Justice; Journal of Research in Crime and Delinquency; Law and Human Behavior; Psychology of Violence; Trauma, Violence and Abuse; and Youth Violence and Juvenile Justice, classified in all thematic categories of the databases (JCR and SJR) in quartile 1. The British Journal of Criminology (Q1 in JCR and Q1 and Q2 in SJR) would also be highly recommended. However, the most frequent (24 of 30 journals), is for a journal to occupy different positions and, therefore, different quartile, depending on the subject category in which it had been classified.

From the total publications analysed, from the point of view of the evaluation criteria of the National Evaluator Commission of the Research Activity (CNEAI), only 18 Journals of forensic psychology, from the ones analyzed in this study, and from those included in the JCR, would be of use for the accreditation of Spanish re- searchers. Journals that meet these requirements are: British Journal of Criminology (Q1), Journal of Criminal Justice (Q1), Journal of Research in Crime and Delinquency (Q1), Law and Human Behaviour (Q1, Q1), Psychology of Violence (Q1, Q1, Q1), Trauma, Violence and Abuse (Q1, Q1, Q1), Youth Violence and Juvenile Justice (Q1), Aggression and Violent Behaviour (Q1, Q2), Legal and Criminological Psychology (Q1) (, Q1, Q2), Sexual Abuse-A Journal of Research and Treatment (Q2, Q1), Behavioural Sciences and the Law (Q2, Q3), Criminal Behaviour and Mental Health (Q2, Q3), Criminal Justice and Behaviour (Q2, Q3), European Journal of Psychology Applied to Legal Context (Q2, Q3), Homicide Studies (Q2), International Journal of Offender Therapy and Comparative Criminology (Q2, Q3), Journal of Interpersonal Violence (Q2, Q2 Q2) Psychology, Crime \& Law (Q2, Q2, Q3).

Analysis of legal psychology journals demonstrates the existence of a discipline which, in Spain, it is getting more and more consolidated everyday but which needs to have more established journals, in order for them to be able to publish the results of their research in national journals of their specialty. Currently, the few options that specialists within the field have to publish papers in journals of their discipline to serve them to recognize their academic merits, contributes to these sending out and publishing their work to journals of general or multidisciplinary nature, which in turn contributes to subtracting visibility to the work and means their work will most likely pass unnoticed for the rest of specialists within the field, which they consult for their training or updating the bibliographic information in journals. 